\title{
Video Article \\ Use of a Wireless Video-EEG System to Monitor Epileptiform Discharges Following Lateral Fluid-Percussion Induced Traumatic Brain Injury
}

\author{
Matthew J. McGuire ${ }^{1}$, Steven M. Gertz ${ }^{1}$, Jolie D. McCutcheon ${ }^{2}$, Chelsea R. Richardson ${ }^{3}$, David J. Poulsen ${ }^{1}$ \\ ${ }^{1}$ Department of Neurosurgery, Jacobs School of Medicine and Biomedical Science \\ ${ }^{2}$ Comparative Medicine Laboratory Animal Facilities, University at Buffalo \\ 3 emka Technologies
}

Correspondence to: David J. Poulsen at davidpou@buffalo.edu

URL: https://www.jove.com/video/59637

DOI: doi:10.3791/59637

Keywords: Behavior, Issue 148, post-traumatic epilepsy, epileptogenesis, lateral fluid-percussion injury, seizure, video-EEG monitoring, wireless telemetry

Date Published: 6/21/2019

Citation: McGuire, M.J., Gertz, S.M., McCutcheon, J.D., Richardson, C.R., Poulsen, D.J. Use of a Wireless Video-EEG System to Monitor Epileptiform Discharges Following Lateral Fluid-Percussion Induced Traumatic Brain Injury. J. Vis. Exp. (148), e59637, doi:10.3791/59637 (2019).

\section{Abstract}

The lateral fluid percussion injury (FPI) model is well established and has been used to study TBI and post-traumatic epilepsy (PTE). However, considerable variability has been reported for the specific parameters used in different studies that have employed this model, making it difficult to harmonize and interpret the results between laboratories. For example, variability has been reported regarding the size and location of the craniectomy, how the Luer lock hub is placed relative to the craniectomy, the atmospheric pressure applied to the dura and the duration of the pressure pulse. Each of these parameters can impact injury severity, which directly correlates with the incidence of PTE. This has been manifested as a wide range of mortality rates, righting reflex times and incidence of convulsive seizures reported. Here we provide a detailed protocol for the method we have used to help facilitate harmonization between studies. We used FPI in combination with a wireless EEG telemetry system to continuously monitor for electrographic changes and detect seizure activity. FPI is induced by creating a $5 \mathrm{~mm}$ craniectomy over the left hemisphere, between the Bregma and Lambda and adjacent to the lateral ridge. A Luer lock hub is secured onto the skull over the craniectomy. This hub is connected to the FPI device, and a 20-millisecond pressure pulse is delivered directly to the intact dura through pressure tubing connected to the hub via a twist lock connector. Following recovery, rats are re-anesthetized to remove the hub. Five $0.5 \mathrm{~mm}$, stainless steel EEG electrode screws are placed in contact with the dura through the skull and serve as four recording electrodes and one reference electrode. The electrode wires are collected into a pedestal connector which is secured into place with bone cement. Continuous video/EEG recordings are collected for up to 4 weeks post TBI.

\section{Video Link}

The video component of this article can be found at https://www.jove.com/video/59637/

\section{Introduction}

In a 2015 report to Congress, the Centers for Disease Control reported that approximately 2.5 million people per year suffer traumatic brain injury (TBI) in the US ${ }^{1}$. It is estimated that TBI causes $20 \%$ of symptomatic epilepsies and $5 \%$ of all epilepsies ${ }^{2,3,4}$. In addition, about $20 \%$ of TBI patients develop post-traumatic epilepsy ${ }^{5}$. Importantly, chronic, recurrent seizures that occur as a consequence of TBI are often pharmacoresistant, increasing the burden of the disease ${ }^{6}$. The exact mechanisms that lead to post-traumatic epilepsy (PTE) remain unclear. However, several key epidemiology studies have examined the incidence and potential risk of developing post-traumatic epilepsy $(\mathrm{PTE})^{2,4,7,8,9,10,11}$. These epidemiology studies each reinforced the correlation of injury severity with the risk of epileptogenesis.

Current methods that have been extensively used to identify novel anti-epilepsy therapies have relied heavily on models that use chemoconvulsants or electrical kindling to induce epilepsy ${ }^{12}$. Given the high incidence of pharmaco-resistance to drugs developed in these models by TBI patients, we hypothesize that TBI-induced seizures may be different from chemoconvulsant or kindling-induced seizures and may involve different pathways or processes of epileptogenesis. Therefore, a TBI model may be better suited for the development of treatments that are more effective to prevent post-traumatic epileptogenesis.

The fluid percussion injury (FPI) model of TBI has been used for decades and is a well-established method to investigate both TBI and PTE $^{13,14,15,16,17,18}$. However, as we recently reviewed, there is a high degree of variability in the FPI methods reported across laboratories ${ }^{19,20}$ This lack of consistency between laboratories prevents reproducibility of preclinical findings and makes the interpretation of results a challenge. As a consequence, increased interest and efforts have been applied towards establishing a greater harmonization for these types of studies $^{21,22,23,24}$.

In an effort to further increase the consistency and harmonization between laboratories focused on studying post-traumatic epileptogenesis, we provide here a detailed methodology of our approach. We have previously reported a $60 \%$ incidence of convulsive seizures within six weeks after severe $\mathrm{TBI}^{20}$. We now use this approach to monitor rats beginning the day of injury and continuously follow them 24 hours a day for up to 
4 weeks. We have chosen to use a wireless telemetry system which affords several advantages. First, rats are able to freely move about their cage, and thus reduces stress. Second a reduction in signal noise as the rat serves as the ground. In addition, our current system employs an accelerometer which detects rapid movement in all three planes (X, $\mathrm{Y}$ and $\mathrm{Z}$ ) and can be helpful to identify convulsive seizure events. Finally, the wireless telemetry system allows for easier management of rats such as supplemental saline injections, weighing and conducting neurological severity scores, which is complicated when rats are attached to a tether. However, this approach also has several limitations. First, the initial cost of a system to record from up to eight rats simultaneously can be in the range of $\$ 60,000$. Second, power is limited by a battery source. This requires daily monitoring and replacement of batteries. The time required between battery changes can be influenced by the sampling rate. However, for a $1000 \mathrm{~Hz}$ sampling rate, batteries are typically changed once a week. The limited power supply also restricts the system to recording from only four EEG signals. Finally, signal drop out is limited but does occasionally occur. However, this approach provides a consistent and reliable method to monitor post-traumatic epileptogenesis and can aid in the identification of novel therapeutic treatments.

\section{Protocol}

All procedures were approved by and followed guidelines of the University at Buffalo Institutional Animal Care and Use committee.

\section{Fluid percussion injury}

1. Wear a lab coat or surgical gown, surgical mask, surgical gloves, and head covering and sterilize all tools and materials that contact the surgical site.

2. Anesthetize a $10-12$-week-old, male, Wistar rat (350-400 g) with $3 \%$ isoflurane and $1 \mathrm{~L} / \mathrm{min}$ oxygen in an induction chamber of appropriate size for rats. Remove the rat from the induction chamber and move it to the prep area once it is unconscious. Put the sterile ophthalmic ointment into both eyes.

3. Shave the hair on the rat's head with electric clippers with a \#40 blade from just above the eyes to the caudal base of the ears to produce enough surgical field. Remove any loose, clipped hair from the site.

4. Clean the surgical site by applying $2 \%$ chlorohexidine scrub to the shaved scalp followed by $70 \%$ ethanol. Start at the center and move outward in concentric circles away from the incision site. Repeat this process 3 times. Apply Betadine solution to the site in the same fashion and allowed to dry.

5. Place the anesthetized rat into the stereotaxic frame and maintain anesthesia at $2-3 \%$ isoflurane- $1 \mathrm{~L} /$ min oxygen via nosecone. Check for loss of withdrawal reflex of hindlimb and loss of palpebral reflex to ensure the rat is in a surgical plane of anesthesia.

6. Monitor the respiratory rate, heart rate, body temperature and oxygen saturation throughout the surgery. Maintain heart rate between $300-400$ bpm, and $\mathrm{SpO}_{2}$ above $90 \%$.

NOTE: A pulse-oximeter attached to a rear foot can be used to provide the constant read out of heart rate and $\mathrm{SpO}_{2}$. $\mathrm{A}$ heart rate above 400 $\mathrm{bpm}$ indicates the rat is not sufficiently anesthetized. A self-regulating warming pad, coupled to a rectal thermometer, set at $37^{\circ} \mathrm{C}$, can be positioned under the rat throughout the surgery to maintain body temperature.A stereomicroscope with a light source in combination with an optic fiber lamp are helpful for visualizing the procedure.

7. Use a 23 g needle to inject $0.5 \%$ bupivacaine hydrochloride intradermally into the scalp at the incision site for local analgesia 10 - 15 minutes prior to making an incision.

8. Make a $1.5-2.5 \mathrm{~cm}$ midline incision through the skin and muscle of the scalp using a \#10 scalpel blade. Retract the skin and muscle to expose the skull and provide a clear surgical field. Reflect the underlying fascia and fatty tissue away from the bone with sterile cotton swabs. NOTE: An electric cautery unit is useful for achieving quick hemostasis.

9. Shave down the lateral ridge of the left parietal bone using a surgical curette to produce a smooth flat surface so that the base of the femalefemale Luer lock hub can rest flush with the skull.

10. Irrigate the skull surface and surrounding tissues with $2.0 \mathrm{mg} / \mathrm{mL}$ gentamicin solution in sterile saline. Blot excess solution with a sterile swabs.

11. Apply $3 \%$ hydrogen peroxide to the skull to dry the bone.

NOTE: If the bone is not sufficiently dry the dental cement will not adhere properly and form a solid seal.

12. Create a $5 \mathrm{~mm}$ diameter craniectomy site through the left parietal bone.

NOTE: A trephine bit placed into a power drill attached to the stereotactic frame can be helpful to initiate the craniectomy. Use a hand drill with a $5 \mathrm{~mm}$ diameter trephine to slowly finish the craniectomy through the remaining bone. When close to completing the craniectomy, rotate the trephine in reverse to prevent rupture of the underlying dura mater. There will be a thinning of the skull around the perimeter of the disk and the skull flap will feel loose when pressed lightly.

13. Remove the bone flap with the surgical curette and smooth tissue forceps. NOTE: Some bleeding may occur, but hemostasis can be quickly achieved by applying gentle pressure with sterile cotton swabs.

14. Use a stereomicroscope and illumination to visually inspect the dura for any signs of rupture. A thin rim of bone will remain around the circumference of the craniectomy site. Gently remove this rim with smooth tissue forceps taking care not to rupture the dura.

15. Swab the skull with $70 \%$ ethanol to remove any bone dust and to dry the skull.

16. Apply a thin layer of cyanoacrylate gel glue around the bottom edge of the Luer lock hub and secure it to the skull over the craniectomy without obstructing the opening. Use caution not to bring the glue in contact with the dura. Further, seal the Luer lock in place with an additional thin layer of glue around the outside base of the hub.

17. Prepare a slurry of dental cement. Apply the cement to the surface of the skull around and over the base of the Luer lock hub to secure it in place.

18. Fill the Luer lock hub with a sterile preservative free artificial cereberal spinal fluid (CSF) solution (pH 7.4) using a syringe and needle so that a convex bolus of saline can be seen above the top of the rim.

NOTE: The solution will keep the dura moist as the dental cement dries as well as serves as an indication of the integrity of the seal. If the solution level falls at all, that is an indication of a leak in the system and the Luer lock must be removed and replaced.

19. Once the dental cement is completely cured, discontinue gas anesthesia and remove the rat from the stereotaxic frame.

20. Place the rat on a platform next to the FPI device. 
21. The FPI device has a curved metal tip that extends from the pressure transducer at the end of the fluid reservoir. Secure a $12 \mathrm{~cm}$ length of pressure tubing to the end of the curved tip with the opposite end terminating in a $2 \mathrm{~cm}$ male Luer lock twist connector. Secure the rat to the FPI device by connecting the female end of the hub on the rat's skull to the male connector. NOTE: Ensure the connection is tightly secured and that all air bubbles have been removed from the system.

22. Place the animal in sternal recumbency and repeatedly check for return of withdrawal reflex. As soon as the rat regains withdrawal reflex but is still sedated, release the pendulum of the FPI device to cause a single $20 \mathrm{~ms}$ pressure pulse and induce injury.

NOTE: It is important to not induce the injury while the animal is deeply anesthetized as this tends to cause increased mortality due to neurogenic-induced pulmonary edema. All devices show variability. However, on the device used for this experiment, a $17^{\circ}$ angle placement of the hammer produces a 2.2 - 2.3 atmospheric pressure pulse. Uninjured, sham animals undergo all of the same procedures with the exception of the actual fluid pulse to the induce injury.

23. Immediately disconnect the rat from the FPI device after injury, place it in sternal recumbency, and provide supplemental oxygen (1 L/min) via a nose cone until spontaneous breathing returns. Apnea is an anticipated consequence of the injury. If necessary, provide periodic manual breaths via a bag valve mask until the rat begins to spontaneously breathe on its own.

NOTE: Typically, apnea lasts less than $2 \mathrm{~min}$. A transient rapid rise in heart rate ( $>500 \mathrm{bpm})$ is observed immediately after the administration of the pressure pulse due to a catecholamine burst. This can be monitored with a pulse oximeter attached to the rat's foot and can serve as a possible indicator that a severe injury has occurred.

24. Monitor the rat continuously and record the time of return of righting reflex (stable ambulation on all four limbs).

25. The magnitude of the atmospheric pressure pulse for each rat should be within \pm 0.05 atmospheres of each other. Confirm that each of the pressure pulse produces a smooth signal on the oscilloscope with consistent amplitude and duration.

NOTE: A noisy signal may indicate air bubbles in the system that must be removed prior to delivering the injury pulse. Atmospheric pressure pulses that produce a severe injury, in this experiment, are those that typically result in animal righting times of 30-60 min. This range of righting times are associated with a mortality rate of approximately $40-50 \%$ ).

26. Administer $10 \mathrm{~mL}$ of prewarmed saline subcutaneously as a supportive care.

27. Return the rat to its home cage and allow it to recover for at least $4 \mathrm{~h}$. NOTE: Increased mortality has been observed when the rats are placed immediately back under anesthesia.

\section{Implantation of cortical EEG electrodes and video-EEG recording}

1. At $4 \mathrm{~h}$ after injury, anesthetize the rat as previously described and place it back into the stereotactic frame to remove the Luer lock hub and dental cement.

NOTE: The hub and cement will easily snap off with moderate pressure. When removing the hub, check carefully for any rupture or damage to the dura. Immediatly euthanize any animal with damage to the dura.

2. Apply a small drop of $0.5 \%$ bupivacaine hydrochloride to the skull in each of the locations where 5 pilot holes are to be drilled (see Figure 1 ).

3. Drill pilot holes through the skull with a hand-held $0.1 \mathrm{~mm}$ drill bit.

4. Secure a stainless-steel electrode screw into each pilot hole at the following locations: a reference screw is placed caudal to the lambda over the cerebellum. Recording electrodes are placed: 1) over the hemisphere ipsilateral and rostral to the craniectomy; 2) over the hemisphere ipsilateral and caudal to the craniectomy; 3 ) over the hemisphere contralateral and rostral to the craniectomy; 4) over the hemisphere contralateral and caudal to the craniectomy.

5. Swab the skull with $70 \%$ ethanol to remove any bone dust.

6. Cover the craniectomy site with a thin layer of sterile bone wax to cover the exposed dura.

7. Connect an electrode array to the 5 EEG electrodes by wrapping the exposed end of a color-coded electrode wire tightly around its designated stainless-steel electrode screw.

NOTE: The opposite ends of each electrode wire is positioned into a specific, designated location within the pedestal connector.

8. Prepare a slurry of bone cement.

9. Collect the electrode wires into a coil underneath the pedestal and secure the wires and pedestal into place with bone cement. Hold the pedestal in position until the bone cement has cured.

NOTE: The bone must be particularly dry and void of any residual blood in order to achieve proper adhesion and prevent premature removal of the transmitter.

10. Attach the wireless transmitter with fresh batteries to the pedestal before removing the animal from the stereotactic frame.

11. Place the animal in its home cage and place the cage in proximity to the receiver and in view of a designated video camera. Initiate video/ EEG recording.

\section{Collection of video-EEG recordings}

1. Prior to collecting EEG signals, do a frequency sweep of the room where rats will be housed for EEG collection to identify any potential interfering frequencies to prevent the collection of EEG recording with any frequency that has background noise.

2. Set all transmitters to specific frequencies that are free of interference.

3. Set the sampling frequency and the input range of each programmable transmitter.

NOTE: This can be done using a smart tool provided by the system manufacturer. Transmitters can sample at a maximum rate of $1000 \mathrm{~Hz}$, and a maximum input range of $\pm 10 \mathrm{mV}$. In this experiment, EEG recordings between $0.5 \mathrm{~Hz}$ to $30 \mathrm{~Hz}$ were analyzed. Therefore, the sample rate was set at $250 \mathrm{~Hz}$. We typically observe Amplitudes of less than $1 \mathrm{mV}$. Therefore, the set input range was at $\pm 2 \mathrm{mV}$.

4. Use EEG collection software provided by the manufacturer to continuously record video-EEG beginning on the day of injury linking each wireless transmitter via a unique frequency to a specific receiver.

NOTE: Each transmitter receiver pair is capable of monitoring 4 monopolar EEG channels, and acceleration in the $X, Y$ and $Z$ planes. EEG data can be written to a storage server. The video data should be saved on a NAS device linked to the storage server. The EEG analysis software synchronizes the video and EEG recording based on the time maintained by the storage server.

5. Use the video collection software to record video of each rat with its own 2 MP resolution camera $(1920 \times 1080)$ configured to record at 30 frames/s. 
NOTE: Each camera has its own infrared illumination for video collection at night.

6. Configure the system to automatically save all video and EEG recordings to a storage server every $24 \mathrm{~h}$. The videos produce rather large files.

\section{Video/EEG analysis}

1. Synchronize the video with each EEG recording at $1 / 10 \mathrm{~s}$ resolution. Do this by using the system manufacturers video/EEG analysis software that creates a metafile with the stamp of the precise time of both of the EEG and the video.

2. Manually screen through EEG recordings to identify index events that define seizure activity.

3. Using the video/EEG analysis software and index EEG events, create a configuration file that uses key parameters (i.e., power in specific frequency bands, the ratio of frequency bands to the total power, acceleration threshold, etc.) to define the characteristics of the potential seizure events.

4. Run the EEG analysis software to identify potential regions of EEG recording that qualify based on the parameters selected in the configuration file.

NOTE: The EEG analysis software allows for automatic seizure detection and highlights regions of interest in the EEG signals and provides FFT power spectrum analysis across the signal.

5. Confirm potential convulsive seizures by using video recordings collected during acquisition, which are synchronized with each rat's respective EEG recordings.

\section{Representative Results}

With this model, we induced severe TBI into adult, male, Wistar rats. Under the conditions we describe here, we typically observe mortality rates of $40-50 \%$, and righting reflex times of $30-60 \mathrm{~min}$ as previously described ${ }^{20}$. We were able to collect video/EEG recordings $24 \mathrm{~h} / \mathrm{day}$ beginning on the day of injury. A diagram showing the location of four monopolar EEG electrodes and a single reference electrode is shown in Figure $1 \mathrm{~A}$. Images which demonstrate the location and appearance of the TBI lesions expected with the conditions described here are shown in Figure 1B-D. Under the conditions described here, we consistently observe delta slowing within the first three days post TBI. Less severely injured rats exhibit unilateral, intermittent delta slowing (Figures 2C-D). In contrast, continuous, bilateral delta slowing is observed after more severe injuries (Figure 3C-D). Some degree of delta slowing was consistently observed in all TBI rats but was not detected in any sham operated (craniectomy only) control rats (Figures 2A-B; 3A-B). Extensive delta slowing was consistently observed during the first three days after injury in most TBI rats. Interestingly, rats typically show pronounced weight loss during the first three days post injury. Non-convulsive seizures are occasionally observed within the first week following TBI (Figure 4 C-D). Clinical seizures, presenting as spike clusters associated with rearing and falling as well as forearm clonus can be observed after 1-week post TBI (Figure 5C-D). Finally, Figure 6 presents representative images of occasional intermittent signal drop out and loss of signal due to battery failure.

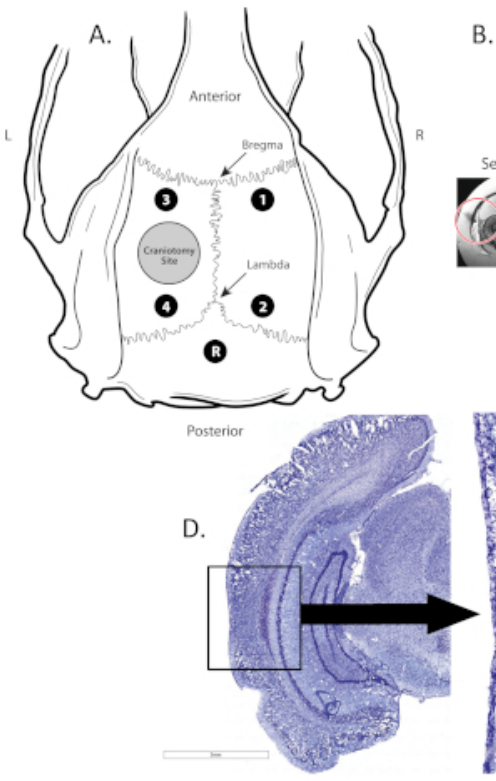
B.

C. Unfolded Cortical Map of

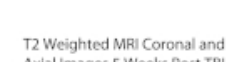

T2 Weighted MRI Coronal and
Axial Images 5 Weeks Post TB

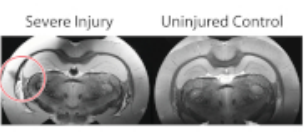
Severe TBl Lesion
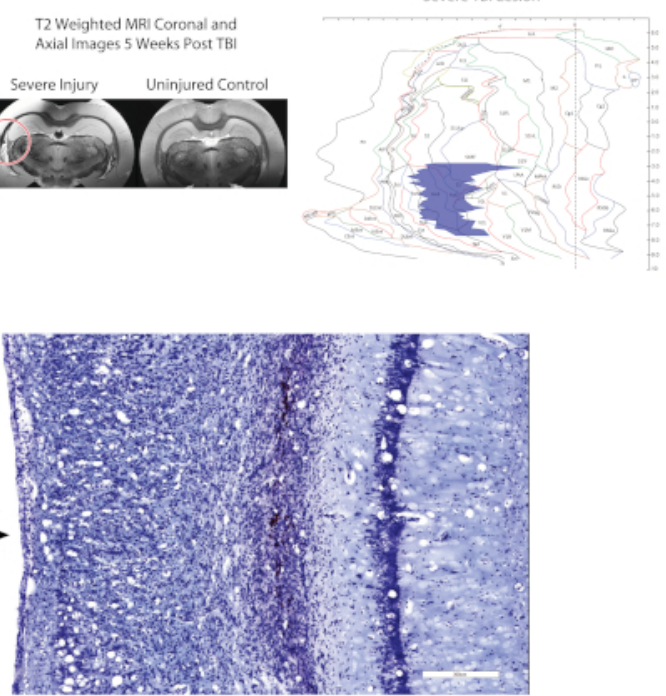

Figure 1. Location of craniectomy, electrode placement, and lesion. (A) shows a schematic diagram of the rat skull with the locations of the craniectomy (grey circle in the left hemisphere), four monopolar electrodes (Black dots; 1,2,3,4) located between the Bregma and Lambda and a reference electrode (Black dot, R) placed midline, posterior to the lambda; (B) shows coronal post-mortem T2 MRI scans with the location of the lesion identified by a red circle; (C) shows a 2-D map of the cortex where the location and size of the lesion is identified (blue region). (D) shows a Nissl stained coronal section with the lesion boxed, lesion is 100x magnified in image to the right. Please click here to view a larger version of this figure. 

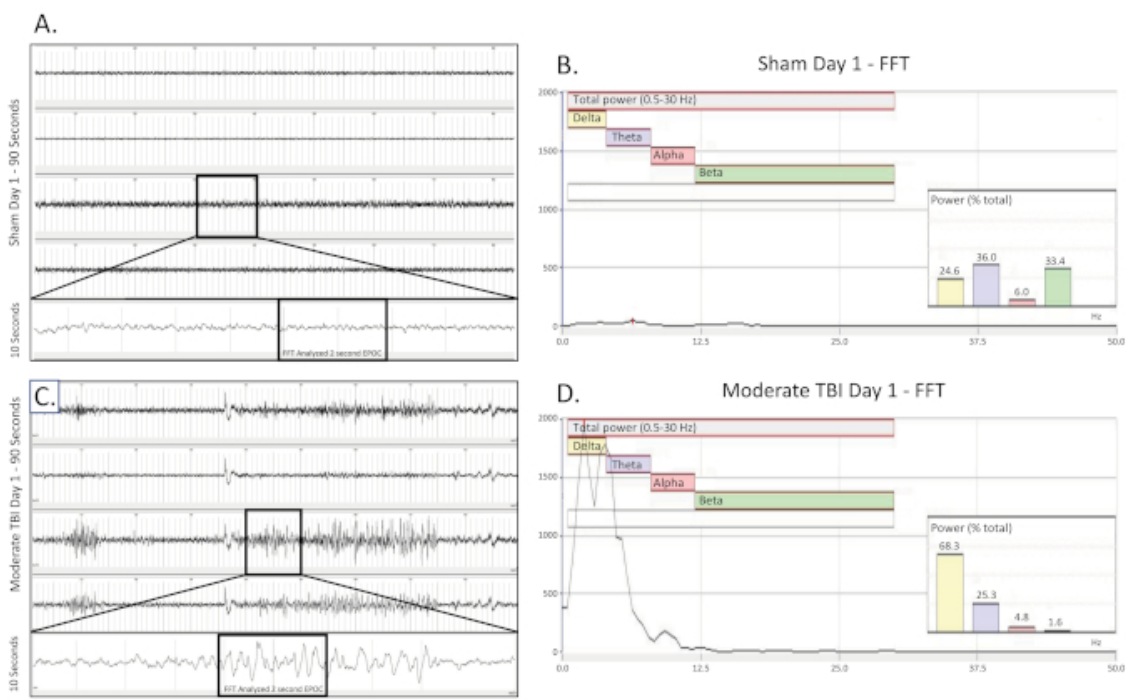

Figure 2. Unilateral, intermittent delta slowing collected on the day of a moderate TBI. (A) shows a $90 \mathrm{~s}$ EEG trace from a sham operated, uninjured control rat on the day of surgery. All four channels are presented. A $10 \mathrm{~s}$ long trace (taken from the boxed region) was extracted from the 3rd channel to better visualize the baseline EEG pattern. A $2048 \mathrm{~ms}$ EPOC section of this was then selected to be analyzed in the corresponding FFT. (B) FFT analysis of $2048 \mathrm{~ms}$ selected EPOC from the uninjured sham operated animal on the day of surgery. (C) shows a $90 \mathrm{~s}$ EEG trace, which demonstrates the intermittent, unilateral delta slowing pattern of a moderately injured animal on the day of injury. A $10 \mathrm{~s}$ long trace (taken from the boxed region) was extracted from the 3rd channel to better visualize the delta slowing EEG pattern. A $2048 \mathrm{~ms}$ EPOC section of this was then selected to be analyzed in the corresponding FFT. (D) FFT analysis of 2048 ms selected EPOC from the moderate TBI animal on the day of injury. 90 s EEG tracings, from top to bottom are biopotentials 1, 2, 3, 4, corresponding to their locations around the craniectomy site as seen in Figure 1. Grey vertical marks define $1 \mathrm{~s}$ intervals on the EEG traces. All EEG traces are shown on a scale of $( \pm 500$ $\mu \mathrm{V})$. Within FFT Analysis graphs, overall analyzed frequency range was $0.5-30 \mathrm{~Hz}$. This was further broken down into 4 separate frequency bands of Delta (Yellow, 0.5-4 Hz), Theta (Purple, 4-8 Hz), Alpha (Red, 8-12 Hz), and Beta (Green, 12-30 Hz). \% (Power) graph shown within the FFT analysis tells what percentage of the total power in the analyzed EPOC comes from each previously specified frequency band, allowing for further mathematical characterization of the EEG waveform patterns. Please click here to view a larger version of this figure. 
A.
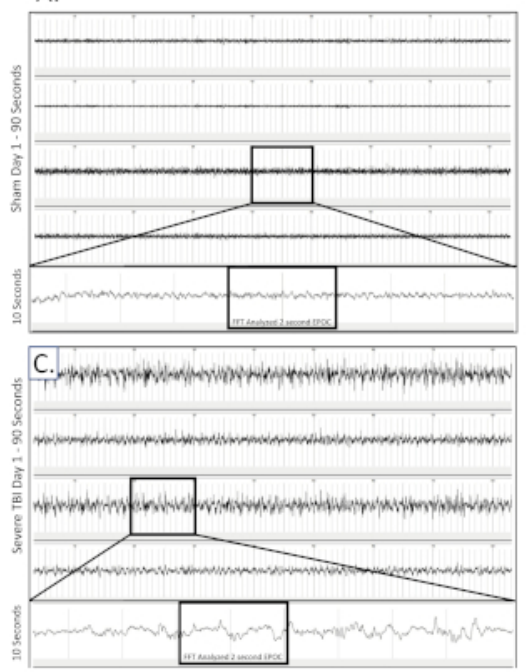

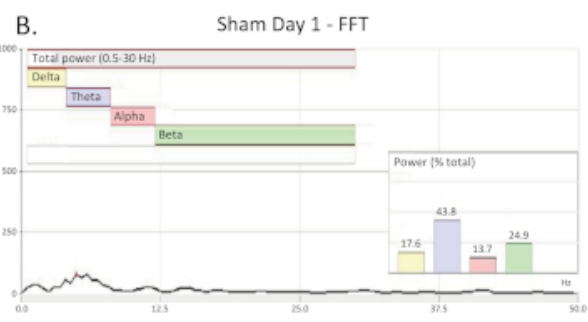

D. Severe TBI Day 1 - FFT

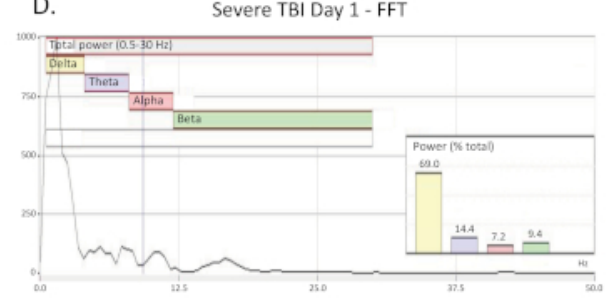

Figure 3. Bilateral, continuous delta slowing collected on the day of a severe TBI. (A) shows a $90 \mathrm{~s}$ EEG trace from a sham operated, uninjured control rat on the day of surgery. All four channels are presented. A $10 \mathrm{~s}$ long trace (taken from the boxed region) was extracted from the 3rd channel to better visualize the baseline EEG pattern. A $2048 \mathrm{~ms}$ EPOC section of this was then selected to be analyzed in the corresponding FFT. (B) FFT analysis of $2048 \mathrm{~ms}$ selected EPOC from the uninjured sham operated animal on the day of surgery. (C) shows a $90 \mathrm{~s}$ EEG trace, which demonstrates the continuous, bilateral delta slowing pattern of a severely injured animal on the day of injury. A 10 $\mathrm{s}$ long trace (taken from the boxed region) was extracted from the 3rd channel to better visualize the delta slowing EEG pattern. A $2048 \mathrm{~ms}$ EPOC section of this was then selected to be analyzed in the corresponding FFT. (D) FFT analysis of 2048 ms selected EPOC from the severe TBI animal on the day of injury. 90 s EEG tracings, from top to bottom are biopotentials 1, 2, 3, 4, corresponding to their locations around the craniectomy site as seen in Figure 1. Grey vertical marks define $1 \mathrm{~s}$ intervals on the EEG traces. All EEG traces are shown on a scale of $( \pm 500$ $\mu \mathrm{V})$. Within FFT Analysis graphs, overall analyzed frequency range was $0.5-30 \mathrm{~Hz}$. This was further broken down into 4 separate frequency bands of Delta (Yellow, 0.5-4 Hz), Theta (Purple, 4-8 Hz), Alpha (Red, 8-12 Hz), and Beta (Green, 12-30 Hz). \% (Power) graph shown within the FFT analysis tells what percentage of the total power in the analyzed EPOC comes from each previously specified frequency band, allowing for further mathematical characterization of the EEG waveform patterns. Please click here to view a larger version of this figure. 
A.

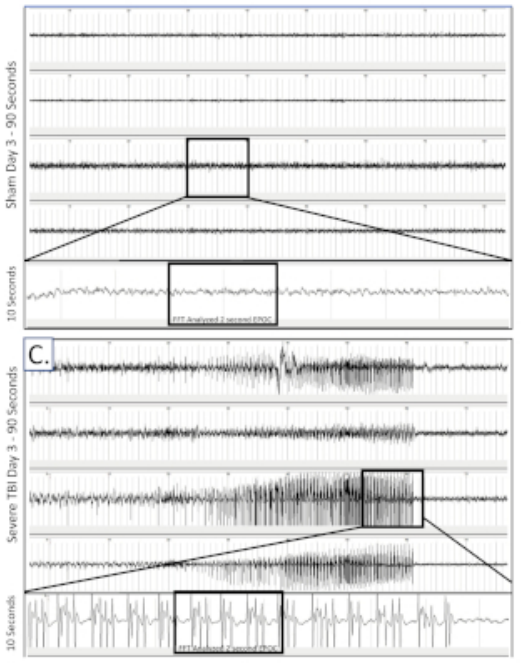

B.

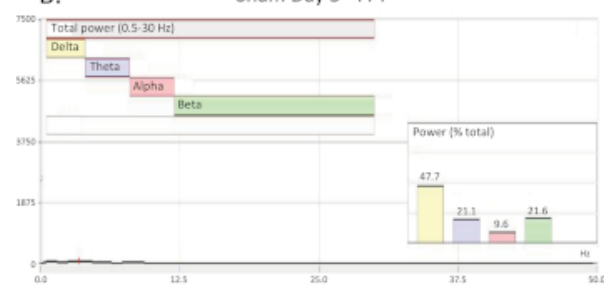

D.

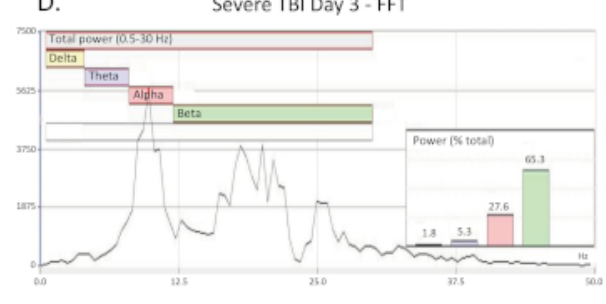

Figure 4. Nonconvulsive electrographic seizure collected 3 days post severe TBI. (A) shows a $90 \mathrm{~s}$ EEG trace from a sham operated, uninjured control rat 3 days ${ }^{25}$ after surgery. All four channels are presented. A $10 \mathrm{~s}$ long trace (taken from the boxed region) was extracted from the 3rd channel to better visualize the baseline EEG pattern. A $2048 \mathrm{~ms}$ EPOC section of this was then selected to be analyzed in the corresponding FFT. (B) FFT analysis of $2048 \mathrm{~ms}$ selected EPOC from the uninjured sham operated animal on the day three ${ }^{25}$ after of surgery. (C) shows a $90 \mathrm{~s}$ EEG trace three ${ }^{25}$ days post severe injury. This show building, fast spiking pattern present bilaterally and across all 4 collecting channels. A $10 \mathrm{~s}$ long trace (taken from the boxed region) was extracted from the 3rd channel to better visualize the spiking EEG pattern. A $2048 \mathrm{~ms}$ EPOC section of this was then selected to be analyzed in the corresponding FFT. (D) FFT analysis of $2048 \mathrm{~ms}$ selected EPOC from the severe TBI animal on the day of injury. 90 s EEG tracings, from top to bottom are biopotentials 1, 2, 3, 4, corresponding to their locations around the craniectomy site as seen in Figure 1. Grey vertical marks define $1 \mathrm{~s}$ intervals on the EEG traces. All EEG traces are shown on a scale of $( \pm 500 \mu \mathrm{V})$. Within FFT Analysis graphs, overall analyzed frequency range was $0.5-30 \mathrm{~Hz}$. This was further broken down into 4 separate frequency bands of Delta (Yellow, 0.5-4 Hz), Theta (Purple, 4-8 Hz), Alpha (Red, 8-12 Hz), and Beta (Green, 12-30 Hz). \% (Power) graph shown within the FFT analysis tells what percentage of the total power in the analyzed EPOC comes from each previously specified frequency band, allowing for further mathematical characterization of the EEG waveform patterns. Please click here to view a larger version of this figure. 
A.
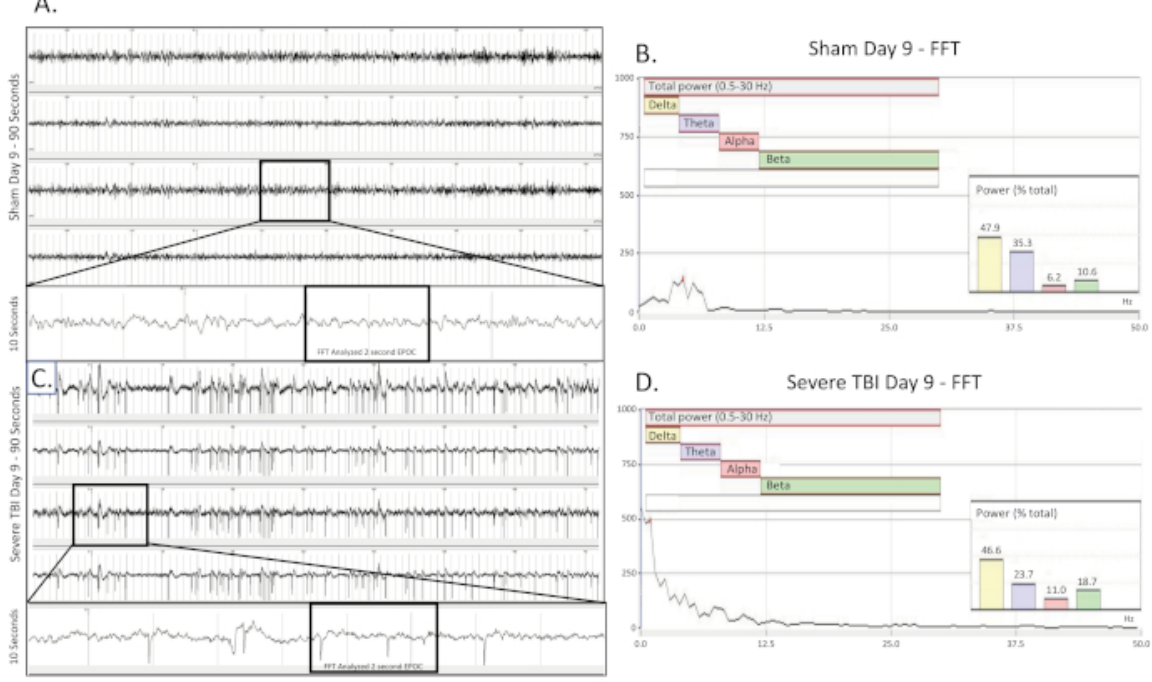

Figure 5. Convulsive electrographic seizure collected 9 days post TBI. (A) shows a $90 \mathrm{~s}$ EEG trace from a sham operated, uninjured control rat nine (9) days after surgery. All four channels are presented. A $10 \mathrm{~s}$ long trace (taken from the boxed region) was extracted from the 3rd channel to better visualize the baseline EEG pattern. A $2048 \mathrm{~ms}$ EPOC section of this was then selected to be analyzed in the corresponding FFT. (B) FFT analysis of $2048 \mathrm{~ms}$ selected EPOC from the uninjured sham operated animal on the day nine (9) after of surgery. (C) shows a 90 $s$ EEG trace nine (9) days post severe injury. This show building, fast spiking pattern present bilaterally and across all 4 collecting channels. A $10 \mathrm{~s}$ long trace (taken from the boxed region) was extracted from the 3rd channel to better visualize the spiking EEG pattern. A $2048 \mathrm{~ms}$ EPOC section of this was then selected to be analyzed in the corresponding FFT. (D) FFT analysis of 2048 ms selected EPOC from the severe TBI animal nine (9) days post injury. 90 s EEG tracings, from top to bottom are biopotentials 1, 2, 3, 4, corresponding to their locations around the craniectomy site as seen in Figure 1. Grey vertical marks define $1 \mathrm{~s}$ intervals on the EEG traces. All EEG traces are shown on a scale of $( \pm 500$ $\mu \mathrm{V})$. Within FFT Analysis graphs, overall analyzed frequency range was $0.5-30 \mathrm{~Hz}$. This was further broken down into 4 separate frequency bands of Delta (Yellow, 0.5-4 Hz), Theta (Purple, 4-8 Hz), Alpha (Red, 8-12 Hz), and Beta (Green, 12-30 Hz). \%(Power) graph shown within the FFT analysis tells what percentage of the total power in the analyzed EPOC comes from each previously specified frequency band, allowing for further mathematical characterization of the EEG waveform patterns. Please click here to view a larger version of this figure.

A. Intermittent Signal Dropout

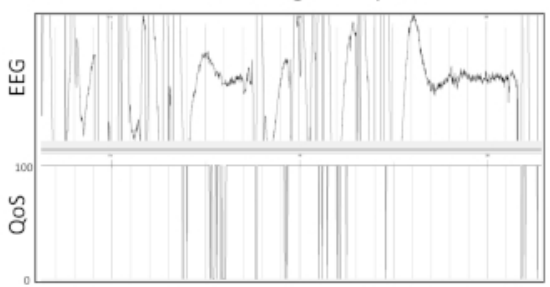

C.

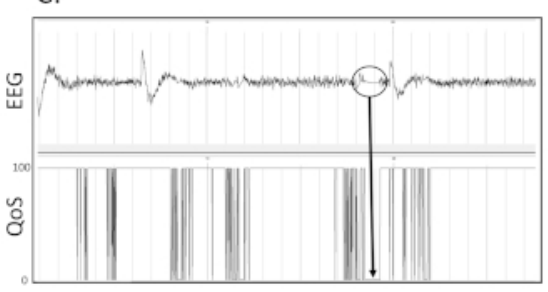

B. Dead Battery Signal Dropout

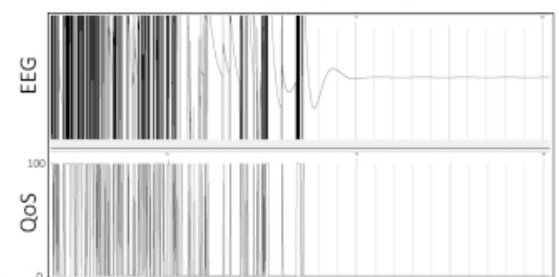

Figure 6. Signal drop out. These are 3 separate examples of what signal drop out due to transmitter or receiver issues appears as on the EEG recording. (A) This is an example of intermittent dropout of the EEG signal on a recording. (B) This is an example of drop out due to battery failure during continuous wireless telemetry appears as on an EEG tracing. (C) Within the circled region, it can be seen that when the Quality of Signal (QoS) drops from 100 to 0 , the EEG tracing becomes flattened and stagnant at $0 \mu \mathrm{V}$. Grey vertical marks define $1 \mathrm{~s}$ intervals on the EEG traces. All EEG traces are shown on a scale of $( \pm 500 \mu \mathrm{V})$. Please click here to view a larger version of this figure.

\section{Discussion}

Considerable variability has been reported between laboratories regarding the specific parameters and methods used for the FPI TBI model $14,26,27,28$. These inconsistencies have resulted in conflicting results and make it difficult to harmonize efforts and outcomes between labs. Here, 
we have presented a detailed methodology describing our approach to long-term, continuous recording of video/EEG to monitor for posttraumatic epileptiform activity. A number of steps are critical to generating reproducible results with the described method.

First, given that the incidence of post-traumatic epilepsy correlates with injury severity, apply conditions that result in the most severe TBI. Specifically, use a $5 \mathrm{~mm}$ craniectomy to ensure that a sufficiently large area of dura is exposed. In addition, secure a female-female Luer lock device onto the surface of the skull, with the opening placed directly over the craniectomy. This differs from other labs that have used a smaller craniectomy $(3 \mathrm{~mm})$ and/or placed a modified needle hub inside the craniectomy, which effectively reduces the opening size. By placing the Luer lock outside of the craniectomy, the $5 \mathrm{~mm}$ opening is maintained. These specific parameters impact the overall force applied to the dura. The atmospheric pressure applied to the dura also has a major impact on the severity of injury observed. Unfortunately, atmospheric pressure is highly variable and appears to be device dependent. Some labs have reported applying a pressure pulse of $8-10 \mathrm{~ms}{ }^{18}$. In contrast, the method described here results in a $20 \mathrm{~ms}$ pressure pulse. This is consistent with other labs that appear to generate more severe injury ${ }^{14,28}$. It is clear that the injury-inducing pressure pulse is a parameter that shows considerable variability between labs and must be empirically defined. However, injury severity may be determined based on a combination of mortality rates (40-50\%), righting reflex times (>30 min) ${ }^{26}$. It is also critical that only animals with an intact dura be included in the study. In addition, if the craniectomy is occluded by any glue or cement such that part of the dura beneath the craniectomy is not exposed to the full force of the fluid pressure pulse, then the animal should be eliminated from the study. Also, excess glue beneath the Luer lock can adhere to the dura and remove it with the cement cap even after a successful injury. Finally, the smooth shape of the pressure pulse curve on the oscilloscope trace gives the indication that there are no air bubbles in the fluid chamber and indicates the plunger is moving without impedance.

Anesthesia is another critical factor that must be controlled. Isoflurane exposure should be kept to the lowest levels possible to maintain a surgical plane of anesthesia. Rats exposed to higher levels of isoflurane or for long durations are more likely to develop neurogenic-induced pulmonary edema. Preparation of the skull represents another critical aspect of the method. Particularly, drying the skull and removing any bone dust helps to prevent the rats from removing the transmitter prematurely.

The placement of screws and the connection of the EEG wires are obviously critical to producing consistently reproducible recordings. It is important that the screws are not placed too deeply as to induce a lesion on the brain. The bone flap recovered from the craniectomy of adult (12 weeks old) male Wistar rats is consistently $2 \mathrm{~mm}$ thick. Use EEG electrode screws with a $2.5 \mathrm{~mm}$ shaft. It is helpful to use the tips of curved mosquito hemostatic forceps as a spacer to ensure that the screws only extend to the base of the bone and do not protrude into the brain.

The approach presented here does have some limitations. Batteries must be changed on a regular basis. The frequency of battery changes depends on the sampling rate. Batteries are typically changed once a week for a sampling rate of $1000 \mathrm{~Hz}$. This time frame can be extended by reducing the sampling rate. The system is also limited to recording from four monopolar EEG electrodes. However, this provides two channels per hemisphere and can differentiate between focal and generalized events and can differentiate between anterior and posterior changes. Despite these limitations, this approach provides a reasonable method to conduct continuous video/EEG monitoring and detection of epileptiform changes following severe TBI.

The method described here results in both electrographic and convulsive seizures within one month following TBI. Therefore, this approach provides a reasonable time frame in which to study potential therapeutics for preventing epileptogenesis following severe TBI. This approach also provides a method to investigate the molecular mechanisms associated with PTE and may lead to the identification of potential biomarkers that can be used to identify patients who are most at risk of developing PTE.

\section{Disclosures}

Chelasea R Richardson is an employee of emka Scientific, the supplier of this wireless telemetry system described.

\section{Acknowledgments}

We wish to thank Paul Dressel for his invaluable support in graphical design and preparation of figures.

\section{References}

1. Flanagan, S.R. Invited Commentary on Centers for Disease Control and Prevention Report to Congress: Traumatic Brain Injury in the United States: Epidemiology and Rehabilitation. Archives of Physical Medicine and Rehabilitation. 96, 1753-1755 (2015).

2. Annegers, J.F., Coan, S.P., Hauser, W.A., Leestma, J., Duffell, W., Tarver, B. Epilepsy, vagal nerve stimulation by the NCP system, mortality, and sudden, unexpected, unexplained death. Epilepsia. 39, 206-212 (1998).

3. Lowenstein, D.H. Epilepsy after head injury: an overview. Epilepsia. 50 Suppl 2, 4-9 (2009).

4. Englander, J., et al. Analyzing risk factors for late posttraumatic seizures: a prospective, multicenter investigation. Archives of Physical Medicine and Rehabilitation. 84, 365-373 (2003).

5. Faul, M.X.L., Wald, M.M., Coronado, V.G. Traumatic Brain Injury in the United States: Emergency Department Visits, Hospitalizations and Deaths 2002-2006. Atlanta (GA): Centers for Disease Control and Prevention, National Center for Injury Prevention and Control. (2010).

6. Herman, S.T. Epilepsy after brain insult: targeting epileptogenesis. Neurology. 59, S21-26 (2002).

7. Annegers, J.F., Coan, S.P. The risks of epilepsy after traumatic brain injury. Seizure. 9:453-457 (2000).

8. Christensen, J., Pedersen, M.G., Pedersen, C.B., Sidenius, P., Olsen, J., Vestergaard, M. Long-term risk of epilepsy after traumatic brain injury in children and young adults: a population-based cohort study. Lancet. 373, 1105-1110 (2009).

9. Webb, T.S., Whitehead, C.R., Wells, T.S., Gore, R.K., Otte, C.N. Neurologically-related sequelae associated with mild traumatic brain injury. Brain Injury. 29, 430-437 (2015).

10. Mahler, B., Carlsson, S., Andersson, T., Adelow, C., Ahlbom, A., Tomson, T. Unprovoked seizures after traumatic brain injury: A populationbased case-control study. Epilepsia. 56, 1438-1444 (2015). 
11. Wang, H., et al. Post-traumatic seizures--a prospective, multicenter, large case study after head injury in China. Epilepsy Research. 107, 272-278 (2013).

12. Simonato, M., French, J.A., Galanopoulou, A.S., O'Brien, T.J. Issues for new antiepilepsy drug development. Current Opinion in Neurology. 26, 195-200 (2013).

13. Xiong, Y., Mahmood, A., Chopp, M. Animal models of traumatic brain injury. Nature Review Neuroscience. 14,128-142 (2013).

14. Kharatishvili, I., Nissinen, J.P., McIntosh, T.K., Pitkanen, A. A model of posttraumatic epilepsy induced by lateral fluid-percussion brain injury in rats. Neuroscienc. 140, 685-697 (2006).

15. Mclntosh, T.K., et al. Traumatic brain injury in the rat: characterization of a lateral fluid-percussion model. Neuroscience. 28, 233-244 (1989).

16. Thompson, H.J., et al. Lateral fluid percussion brain injury: a 15-year review and evaluation. Journal of Neurotrauma. 22, 42-75 (2005).

17. Curia, G., Eastman, C.L., Miller, J.W., D'Ambrosio, R. Modeling Post-Traumatic Epilepsy for Therapy Development. In: Laskowitz D, Grant G, eds. Translational Research in Traumatic Brain Injury. Boca Raton (FL) (2016).

18. D'Ambrosio, R, Fairbanks, J.P., Fender, J.S., Born, D.E., Doyle, D.L., Miller, J.W. Post-traumatic epilepsy following fluid percussion injury in the rat. Brain. 127:304-314 (2004).

19. Saatman, K.E., et al. Classification of traumatic brain injury for targeted therapies. Journal of Neurotrauma. 25, 719-738 (2008).

20. Smith, D., Brooke, D, Wohlgehagen, E., Rau, T., Poulsen, D. Temporal and Spatial Changes in the Pattern of Iba1 and CD68 Staining in the Rat Brain Following Severe Traumatic Brain Injury. Modern Research in Inflammation. 4, 9-23 (2015).

21. Ndode-Ekane, X.E., et al. Harmonization of lateral fluid-percussion injury model production and post-injury monitoring in a preclinical multicenter biomarker discovery study on post-traumatic epileptogenesis. Epilepsy Research. 151, 7-16 (2019).

22. Ciszek, R., et al. Informatics tools to assess the success of procedural harmonization in preclinical multicenter biomarker discovery study on post-traumatic epileptogenesis. Epilepsy Research. 150, 17-26 (2019).

23. Immonen, R., et al. Harmonization of pipeline for preclinical multicenter MRI biomarker discovery in a rat model of post-traumatic epileptogenesis. Epilepsy Research. 150, 46-57 (2019).

24. Kamnaksh, A., et al. Harmonization of pipeline for preclinical multicenter plasma protein and miRNA biomarker discovery in a rat model of post-traumatic epileptogenesis. Epilepsy Research. 149, 92-101 (2019).

25. Redell, J.B., Moore, A.N., Ward, N.H., 3rd, Hergenroeder, G.W., Dash, P.K. Human traumatic brain injury alters plasma microRNA levels. Journal of Neurotrauma. 27, 2147-2156 (2010).

26. Smith, D., et al. Convulsive seizures and EEG spikes after lateral fluid-percussion injury in the rat. Epilepsy Research.147, 87-94 (2018).

27. Eastman, C.L., Fender, J.S., Temkin, N.R., D'Ambrosio, R. Optimized methods for epilepsy therapy development using an etiologically realistic model of focal epilepsy in the rat. Experimental Neurology. 264, 150-162 (2015).

28. Shultz, S.R., et al. Can structural or functional changes following traumatic brain injury in the rat predict epileptic outcome? Epilepsia. 54 1240-1250 (2013). 